Pacific

Journal of

Mathematics

MANIFOLDS WITH POSITIVE SECOND GAUSS-BONNET CURVATURE

MOHAMMED-LARBI LABBI

Volume $227 \quad$ No. 2

October 2006 


\title{
MANIFOLDS WITH POSITIVE SECOND GAUSS-BONNET CURVATURE
}

\author{
MOHAMMED-LARBI LABBI
}

\begin{abstract}
The second Gauss-Bonnet curvature of a Riemannian manifold, denoted $h_{4}$, is a generalization of the four-dimensional Gauss-Bonnet integrand to higher dimensions. It coincides with the second curvature invariant, which appears in the well known Weyl's tube formula. A crucial property of $\boldsymbol{h}_{\mathbf{4}}$ is that it is nonnegative for Einstein manifolds; hence it provides, independently of the sign of the Einstein constant, a geometric obstruction to the existence of Einstein metrics in dimensions $\geq 4$. This motivates our study of the positivity of this invariant. We show that positive sectional curvature implies the positivity of $h_{4}$, and so does positive isotropic curvature in dimensions $\geq 8$. Also, we prove many constructions of metrics with positive second Gauss-Bonnet curvature that generalize similar well known results for the scalar curvature.
\end{abstract}

\section{Introduction and statement of the results}

Let $(M, g)$ be a smooth Riemannian manifold of dimension $n \geq 4$. Let $R, c R$ and $c^{2} R$ denote respectively the Riemann curvature tensor, Ricci tensor and the scalar curvature of $(M, g)$. The second Gauss-Bonnet curvature, which throughout this paper is abbreviated as SGBC and denoted by $h_{4}$, is a generalization of the four-dimensional Gauss-Bonnet integrand to higher dimensions. It is a scalar expression that is quadratic in the curvature tensor. It can be defined by

$$
h_{4}=\|R\|^{2}-\|c R\|^{2}+\frac{1}{4}\left\|c^{2} R\right\|^{2} .
$$

A crucial property of $h_{4}$ is that it is nonnegative for Einstein manifolds (see Section 3 below), and so it provides a new geometric obstruction to the existence of Einstein metrics independently of the sign of the Einstein constant. In particular, the manifolds that do not admit any metric with positive SGBC cannot admit any Einstein metric.

MSC2000: 53C21, 53B20.

Keywords: Gauss-Bonnet curvature, Einstein manifold, surgery.

The author thanks the referee for useful comments and for remarks about terminology on the first version of this paper. 
Recall that in dimensions greater than 4, we do not know any topological restriction for a manifold to be Einstein. If one requires the Einstein constant to be positive, then one has two geometric obstructions $c R>0$ and $c^{2} R>0$.

It would then be a great benefit to have a classification of manifolds with positive SGBC.

In this paper, we inaugurate the study of the positivity properties of this important invariant.

The paper is divided into five sections. In Section 2, we use the ring of curvature structures to introduce and study all of the Gauss-Bonnet curvatures $\left(h_{2 k}\right)$. They are, up to a constant, the curvature invariants that appear in Weyl's tube formula. Many examples are included.

In Section 3, we study separately the case of the second invariant. We prove that it is nonnegative for Einstein manifolds, and nonpositive for conformally flat manifolds with zero scalar curvature. The limit cases are discussed. Also, we prove the following theorem:

Theorem A. Let $(M, g)$ be a Riemannian manifold of dimension $n \geq 4$, with nonnegative $p$-curvature, such that $p \geq n / 2$. Then the $\operatorname{SGBC}$ of $(M, g)$ is nonnegative. Furthermore, it vanishes if and only if the manifold is flat. The same statements hold with "nonnegative" replaced by "positive".

In particular, positive sectional curvature implies positive $S G B C$. Also, if $n \geq 8$, positive isotropic curvature implies positive $S G B C$. The same statements hold with "positive" replaced by "nonnegative".

Therefore we can apply our previous constructions in the class of manifolds with positive $p$-curvature (see Labbi [1997a; 1997b; 2000]) to get many examples of metrics with positive SGBC.

In Section 4, we prove the following useful theorem, which generalizes a similar result for the scalar curvature.

Theorem B. Suppose that the total space $M$ of a Riemannian submersion is compact, and the fibers (with the induced metric) have positive SGBC. Then the manifold $M$ admits a Riemannian metric with positive $S G B C$.

We end the section with two applications of this theorem.

In Section 5, we prove the following stability theorem in the class of compact manifolds with positive SGBC:

Theorem C. If a manifold $M$ is obtained from a compact manifold $X$ by surgery in codimension $\geq 5$, and $X$ admits a metric of positive $S G B C$, then so does $M$.

In particular, the connected sum of two compact manifolds of dimensions $\geq 5$, each one having positive $S G B C$, admits a metric with positive $S G B C$. 
This generalizes a celebrated theorem of Gromov-Lawson and Schoen-Yau for the scalar curvature.

As a consequence of Theorem $\mathrm{C}$, we prove that there are no restrictions on the fundamental group of a compact manifold of dimension $\geq 6$ to carry a metric with positive SGBC.

Finally, we mention that it would be interesting to prove, as in the case of the scalar curvature, that every manifold with nonnegative SGBC not identically zero admits a metric with positive SGBC.

\section{The Gauss-Bonnet curvatures}

The Gauss-Bonnet curvatures are conveniently manipulated with the use of the ring of curvature structures. Let us first recall some properties of this ring.

Let $\wedge^{*} M=\bigoplus_{p \geq 0} \wedge^{* p} M$ denote the ring of differential forms on $M$, where $M$ is as above. Considering the tensor product over the ring of smooth functions, we define $\mathscr{D}=\bigwedge^{*} M \otimes \bigwedge^{*} M=\bigoplus_{p, q \geq 0} \mathscr{D}^{p, q}$, where $\mathscr{D}^{p, q}=\bigwedge^{* p} M \otimes \bigwedge^{* q} M$. It is a graded associative ring and called the ring of double forms on $M$. The KulkarniNomizu product in $\mathscr{D}$ shall be denoted by a dot, omitted whenever possible.

The ring of curvature structures on $M$ [Kulkarni 1972] is the ring $\mathscr{C}=\sum_{p \geq 0} \mathscr{C}^{p}$, where $\mathscr{C}^{p}$ denotes symmetric elements in $\mathscr{D}^{p, p}$. We denote by $\mathscr{C}_{1}, \mathscr{C}_{2}, \mathscr{C}_{0}$ the subring of curvature structures satisfying, respectively, the first, the second, and both the first and second Bianchi identity.

The standard inner product and the Hodge star operator $*$ on $\bigwedge^{* p} M$ can be extended in a standard way to $\mathscr{D}$. These extensions were used in [Labbi 2005] to prove many properties of the former ring. In particular, it is proved that

$$
g \omega=* c * \omega
$$

for all $\omega \in \mathscr{D}$, where c denotes the contraction map. Also, for all $\omega_{1}, \omega_{2} \in \mathscr{D}$, we have

$$
\left\langle g \omega_{1}, \omega_{2}\right\rangle=\left\langle\omega_{1}, c \omega_{2}\right\rangle ;
$$

that is, the contraction map is the formal adjoint of the multiplication map by the metric $g$. We have

$$
\left\langle\omega_{1}, \omega_{2}\right\rangle=*\left(\omega_{1} * \omega_{2}\right)=*\left(* \omega_{1} . \omega_{2}\right),
$$

for all $\omega_{1}, \omega_{2} \in \mathscr{D}^{p, q}$, and

$$
* *=(-1)^{(p+q)(n-p-q)} \mathrm{Id},
$$

where Id is the identity map on $\mathscr{D}^{p, q}$.

Next, we define the Gauss-Bonnet curvatures: 
Definition. The $2 q$-Gauss-Bonnet curvature, denoted $h_{2 q}$, is the complete contraction of the tensor $R^{q}$, precisely,

$$
h_{2 q}=\frac{1}{(2 q) !} c^{2 q} R^{q},
$$

where $R^{q}$ denotes the multiplication of $R$ with itself $q$ times in the ring $\mathscr{b}$.

Note that $h_{2}=\frac{1}{2} c^{2} R$ is one half of the scalar curvature, and if $n$ is even, then $h_{n}$ is (up to a constant) the Gauss-Bonnet integrand.

Finally, note that in [Labbi 2005], it is proved that

$$
h_{2 q}=* \frac{1}{(n-2 q) !} g^{n-2 q} R^{q} .
$$

Example 2.1. Let $(M, g)$ have constant sectional curvature $\lambda$. Then

$$
R=\frac{1}{2} \lambda g^{2} \quad \text { and } \quad R^{q}=\frac{\lambda^{q}}{2^{q}} g^{2 q} .
$$

Therefore, $h_{2 q}$ is constant:

$$
h_{2 q}=* \frac{1}{(n-2 q) !} g^{n-2 q} R^{q}=* \frac{\lambda^{q}}{2^{q}(n-2 q) !} g^{n}=\frac{\lambda^{q} n !}{2^{q}(n-2 q) !} .
$$

In particular,

$$
h_{4}=\frac{n(n-1)(n-2)(n-3)}{4} \lambda^{2} .
$$

Example 2.2. Let $(M, g)$ be a Riemannian product of two Riemannian manifolds $\left(M_{1}, g_{1}\right)$ and $\left(M_{2}, g_{2}\right)$. If we index by $i$ the invariants of the metric $g_{i}$, for $i=1,2$, then

$$
R=R_{1}+R_{2} \quad \text { and } \quad R^{q}=\left(R_{1}+R_{2}\right)^{q}=\sum_{i=0}^{q} C_{i}^{q} R_{1}^{i} R_{2}^{q-i} .
$$

A straightforward calculation then shows that

$$
\begin{aligned}
h_{2 q} & =\frac{c^{2 q} R^{q}}{(2 q) !}=\sum_{i=0}^{q} C_{i}^{q} \frac{c^{2 q}}{(2 q) !}\left(R_{1}^{i} R_{2}^{q-i}\right) \\
& =\sum_{i=0}^{q} C_{i}^{q} \frac{c^{2 i} R_{1}^{i}}{(2 i) !} \frac{c^{2 q-2 i} R_{2}^{q-i}}{(2 q-2 i) !}=\sum_{i=0}^{q} C_{i}^{q}\left(h_{2 i}\right)_{1}\left(h_{2 q-2 i}\right)_{2} .
\end{aligned}
$$

In particular,

$$
h_{4}=\left(h_{4}\right)_{1}+\frac{1}{2} \mathrm{scal}_{1} \mathrm{scal}_{2}+\left(h_{4}\right)_{2} \text {, }
$$

where scal denotes the scalar curvature. 
Example 2.3. Let $(M, g)$ be a hypersurface of the Euclidean space. If $B$ denotes the second fundamental form at a given point, then the Gauss equation shows that

$$
R=\frac{1}{2} B^{2} \quad \text { and } \quad R^{q}=\frac{1}{2^{q}} B^{2 q} .
$$

Consequently, if $\lambda_{1} \leq \lambda_{2} \leq \cdots \leq \lambda_{n}$ denote the eigenvalues of $B$, then the eigenvalues of $R^{q}$ are $\left((2 q) ! / 2^{q}\right) \lambda_{i_{1}}, \lambda_{i_{2}}, \ldots, \lambda_{i_{2 q}}$, where $i_{1}<\cdots<i_{2 q}$. Hence,

$$
h_{2 q}=\frac{(2 q) !}{2^{q}} \sum_{1 \leq i_{1}<\cdots<i_{2 q} \leq n} \lambda_{i_{1}} \ldots \lambda_{i_{2 q}} .
$$

So the Gauss-Bonnet curvatures coincide, up to a constant, with the symmetric functions in the eigenvalues of $B$.

Example 2.4. Let $(M, g)$ be a conformally flat manifold. Then it is well known that, at each point of $M$, the Riemann curvature tensor is determined by a symmetric bilinear form $h$, in the sense that $R=g h$. Hence, $R^{q}=g^{q} h^{q}$.

Let $\left\{e_{1}, \ldots, e_{n}\right\}$ be an orthonormal basis of eigenvectors of $h$, and denote by $\lambda_{1} \leq \lambda_{2} \leq \cdots \leq \lambda_{n}$ the eigenvalues of $h$.

Then it is not difficult to see that, for each $q$, the tensor $R^{q}$ is also diagonalizable by the $2 q$-vectors $e_{i_{1}} \wedge \ldots \wedge e_{i_{2 q}}$, where $i_{1}<\cdots<i_{2 q}$. The eigenvalues are of the form

$$
R^{q}\left(e_{1} \wedge \ldots \wedge e_{2 q}, e_{1} \wedge \ldots \wedge e_{2 q}\right)=(q !)^{2} \sum_{1 \leq i_{1}<\cdots<i_{q} \leq 2 q} \lambda_{i_{1}} \ldots \lambda_{i_{q}} .
$$

Consequently, we get

$$
h_{2 q}=\frac{(n-q) ! q !}{(n-2 q) !} \sum_{1 \leq i_{1}<\cdots<i_{q} \leq n} \lambda_{i_{1}} \ldots \lambda_{i_{q}} .
$$

Example 2.5. Let $g_{t}=t g$ for $t>0$. If we index by $t$ the invariants of $g_{t}$, then

$$
R_{t}=t R \quad \text { and } \quad R_{t}^{q}=t^{q} R^{q},
$$

and therefore,

$$
\left(h_{2 q}\right)_{t}=\frac{1}{t^{q}} h_{2 q} .
$$

We now recall some other useful facts from [Labbi 2005], which shall be used later. Following Kulkarni, we call the elements in $\operatorname{ker} c \subset D^{p, q}$ effective elements of $D^{p, q}$, which shall be denoted by $E^{p, q}$. We have the orthogonal decomposition

$$
D^{p, q}=E^{p, q} \oplus g E^{p-1, q-1} \oplus g^{2} E^{p-2, q-2} \oplus \cdots \oplus g^{r} E^{p-r, q-r},
$$

where $r=\min \{p, q\}$. 
With respect to the previous decomposition, if $\omega=\sum_{i=0}^{p} g^{p-i} \omega_{i} \in \mathscr{C}_{1}^{p}$ and $n=2 p$, then

$$
* \omega=\sum_{i=0}^{p}(-1)^{i} g^{p-i} \omega_{i} .
$$

Lemma 2.6 [Labbi 2005]. Let $\omega_{1} \in E_{1}^{r}, \omega_{2} \in E_{1}^{s}$ be effective. Then

$$
\begin{aligned}
& \left\langle g^{p} \omega_{1}, g^{q} \omega_{2}\right\rangle=0, \quad \text { if } \quad p \neq q \text { or }(p=q \text { and } r \neq s), \\
& \left\langle g^{p} \omega_{1}, g^{p} \omega_{2}\right\rangle=p !\left(\prod_{i=0}^{p-1}(n-2 r-i)\right)\left\langle\omega_{1}, \omega_{2}\right\rangle \quad \text { if } p \geq 1 \text { and } r=s .
\end{aligned}
$$

\section{The second Gauss-Bonnet curvature}

With respect to the orthogonal decomposition (4), the Riemann curvature tensor decomposes to $R=\omega_{2}+g \omega_{1}+g^{2} \omega_{0}$, where

$$
\omega_{0}=\frac{1}{2 n(n-1)} c^{2} R, \quad \omega_{1}=\frac{1}{n-2}\left(c R-\frac{1}{n} g c^{2} R\right),
$$

and $\omega_{2}$ is the Weyl tensor. The latter may be defined by the previous decomposition of $R$.

Corollary 6.5 in [Labbi 2005] shows that

$$
h_{4}=\frac{1}{(n-4) !}\left(n !\left\|\omega_{0}\right\|^{2}-(n-2) !\left\|\omega_{1}\right\|^{2}+(n-4) !\left\|\omega_{2}\right\|^{2}\right) .
$$

Using Lemma 2.6, one can easily check that

$$
\begin{aligned}
\left\|\omega_{2}\right\|^{2} & =\|R\|^{2}-\frac{1}{n-2}\|c R\|^{2}+\frac{1}{2(n-1)(n-2)}\left\|c^{2} R\right\|^{2}, \\
\left\|\omega_{1}\right\|^{2} & =\frac{1}{(n-2)^{2}}\left(\|c R\|^{2}-\frac{1}{n}\left\|c^{2} R\right\|^{2}\right), \\
\left\|\omega_{0}\right\|^{2} & =\frac{1}{4 n^{2}(n-1)^{2}}\left\|c^{2} R\right\|^{2} .
\end{aligned}
$$

Using (6), we obtain another useful expression:

$$
h_{4}=\|R\|^{2}-\|c R\|^{2}+\frac{1}{4}\left\|c^{2} R\right\|^{2} .
$$

The following theorem was first proved in [Labbi 2005] as a special case of a more general result:

Theorem 3.1. Let $(M, g)$ be a Riemannian manifold of dimension $\geq 4$.

(1) If $(M, g)$ is an Einstein manifold, then $h_{4} \geq 0$. If furthermore $h_{4} \equiv 0$, then $(M, g)$ is flat. 
(2) If $(M, g)$ is conformally flat with zero scalar curvature, then $h_{4} \leq 0$. If furthermore $h_{4} \equiv 0$, then $(M, g)$ is flat.

Proof. If $(M, g)$ is conformally flat, then $\omega_{2}=0$, and hence

$$
h_{4}=\frac{1}{(n-4) !}\left(n !\left\|\omega_{0}\right\|^{2}-(n-2) !\left\|\omega_{1}\right\|^{2}\right)=\frac{n-3}{n-2}\left(\frac{n}{4(n-1)}\left\|c^{2} R\right\|^{2}-\|c R\|^{2}\right),
$$

from which it is clear that $c^{2} R=0$ implies $h_{4} \leq 0$. If furthermore $h_{4} \equiv 0$, then the metric is Ricci flat, and hence flat. This proves the first part of the theorem.

Next, if $(M, g)$ is Einstein, then $\omega_{1}=0$, and hence

$$
h_{4}=\frac{1}{(n-4) !}\left(n !\left\|\omega_{0}\right\|^{2}+(n-4) !\left\|\omega_{2}\right\|^{2}\right)=\|R\|^{2}+\frac{n-4}{4 n}\left(c^{2} R\right)^{2},
$$

from which it is clear that $h_{4} \geq 0$. If furthermore $h_{4} \equiv 0$, then the metric is flat.

Recall from [Labbi 1997a; 1997b] that the $p$-curvature of $(M, g)$, denoted by $s_{p}$, for $1 \leq p \leq n-2$, is a function defined on the $p$-Grassmanian bundle of the manifold. Its value at a tangent $p$-plane $P$ is the average of the sectional curvatures of all 2-planes orthogonal to $P$. In particular $s_{0}$ is the scalar curvature, and $s_{n-2}$ is twice the sectional curvature.

Note that the $p$-curvature $s_{p}$ is the sectional curvature of the tensor

$$
\frac{1}{(n-p-2) !} *\left(g^{n-p-2} R\right) \text {. }
$$

The next theorem provides a relation between the positivity of the $p$-curvature and the SGBC.

Theorem A. Let $(M, g)$ be a Riemannian manifold of dimension $n \geq 4$, with nonnegative $p$-curvature, such that $p \geq n / 2$. Then the $\operatorname{SGBC}$ of $(M, g)$ is nonnegative. Furthermore, it vanishes if and only if the manifold is flat. The same statements hold with "nonnegative" replaced by "positive".

Proof. Suppose $n=2(k+2)$ is even, for $k \geq 0$. Since

$$
R=\omega_{2}+g \omega_{1}+g^{2} \omega_{0},
$$

then

$$
g^{k} R=g^{k} \omega_{2}+g^{k+1} \omega_{1}+g^{k+2} \omega_{0} .
$$

Formula (5) shows that

$$
* g^{k} R=g^{k} \omega_{2}-g^{k+1} \omega_{1}+g^{k+2} \omega_{0} .
$$

On the other hand, since $s_{k+2} \geq 0$, both of the tensors $g^{k} R$ and $* g^{k} R$ have positive sectional curvature, hence $\left(g^{k} \omega_{2}+g^{k+2} \omega_{0}\right)\left(e_{i_{1}}, \ldots, e_{i_{k+2}}, e_{i_{1}}, \ldots, e_{i_{k+2}}\right) \geq g^{k+1} \omega_{1}\left(e_{i_{1}}, \ldots, e_{i_{k+2}}, e_{i_{1}}, \ldots, e_{i_{k+2}}\right)$, 
and

$\left(g^{k} \omega_{2}+g^{k+2} \omega_{0}\right)\left(e_{i_{1}}, \ldots, e_{i_{k+2}}, e_{i_{1}}, \ldots, e_{i_{k+2}}\right) \geq-g^{k+1} \omega_{1}\left(e_{i_{1}}, \ldots, e_{i_{k+2}}, e_{i_{1}}, \ldots, e_{i_{k+2}}\right)$,

for all orthonormal vectors $e_{i_{1}}, \ldots, e_{i_{k+2}}$. Therefore

$\left(g^{k} \omega_{2}+g^{k+2} \omega_{0}\right)\left(e_{i_{1}}, \ldots, e_{i_{k+2}}, e_{i_{1}}, \ldots, e_{i_{k+2}}\right) \geq\left|g^{k+1} \omega_{1}\left(e_{i_{1}}, \ldots, e_{i_{k+2}}, e_{i_{1}}, \ldots, e_{i_{k+2}}\right)\right|$.

Using formulas (2) and (1), we get

$$
h_{4}=* \frac{1}{(n-4) !} g^{n-4} R^{2}=* \frac{1}{(2 k) !}\left(g^{k} R \cdot g^{k} R\right)=\frac{1}{(2 k) !}\left\langle g^{k} R, * g^{k} R\right\rangle .
$$

Hence, using Lemma 2.6 and considering an orthonormal basis diagonalizing $c R$, we obtain

$$
\begin{aligned}
(2 k) ! h_{4} & =\left\langle g^{k} \omega_{2}+g^{k+2} \omega_{0}, g^{k} \omega_{2}+g^{k+2} \omega_{0}\right\rangle-\left\langle g^{k+1} \omega_{1}, g^{k+1} \omega_{1}\right\rangle \\
& \geq \sum_{i_{1}<\cdots<i_{k+2}}\left(\left(g^{k} \omega_{2}+g^{k+2} \omega_{0}\right)\left(e_{i_{1}}, \ldots, e_{i_{k+2}}, e_{i_{1}}, \ldots, e_{i_{k+2}}\right)\right)^{2}-\left\|g^{k+1} \omega_{1}\right\|^{2} \\
& \geq \sum_{i_{1}<\cdots<i_{k+2}}\left(g^{k+1} \omega_{1}\left(e_{i_{1}}, \ldots, e_{i_{k+2}}, e_{i_{1}}, \ldots, e_{i_{k+2}}\right)\right)^{2}-\left\|g^{k+1} \omega_{1}\right\|^{2}=0 .
\end{aligned}
$$

The same proof works for strict inequality. Also it is clear that if $s_{k+2} \geq 0$ and $h_{4} \equiv 0$, then $s_{k+2} \equiv 0$, so that the metric is flat.

To complete the proof, note that if the dimension of the manifold $n=2 p+1 \geq 5$ is odd, one can consider the product $M \times S^{1}$. It is of even dimension $2(p+1)$ and has nonnegative or positive $(p+1)$-curvature, as the case may be. Therefore, by (3), the curvature $h_{4}(M)=h_{4}\left(M \times S^{1}\right)$ is nonnegative (positive).

Corollary 3.2. (1) A Riemannian manifold of dimension $\geq 4$ with nonnegative sectional curvature has nonnegative $S G B C$. If furthermore $h_{4} \equiv 0$, the metric is flat.

(2) A Riemannian manifold of dimension $\geq 8$ with nonnegative isotropic curvature has nonnegative SGBC. the metric is flat.

The same statements hold with "nonnegative" replaced by "positive".

Proof. Positive sectional curvature implies positive $p$-curvature, and positive isotropic curvature implies the positivity of the $p$-curvature for all $p \leq n-4$; see [Labbi 2000].

Remarks. 1. If the dimension $n$ of the manifold is even, the algebraic Hopf conjecture states that the positivity of the sectional curvature implies the positivity of the Gauss-Bonnet integrand, that is, $h_{n}>0$. Then one can ask, more generally: Does positive sectional curvature imply the positivity of $h_{2 k}$ for all $2 \leq 2 k \leq n$ ? 
Theorem A shows this is true for $k=1$ and $k=2$. The question remains open for $k \geq 3$.

2. Theorem A generalizes a result of Thorpe [1969] for the dimension $n=4$.

By Corollary 3.2, Lie groups with a biinvariant metric and normal homogeneous Riemannian manifolds have nonnegative SGBC. Also, using Theorem A and our previous results on the $p$-curvature [Labbi 1997a; 1997b], we can easily prove two corollaries:

Corollary 3.3. 1. Let $G$ be a compact connected Lie group with rank $r<$ $\left[\frac{1}{2}(\operatorname{dim} G+1)\right]$ (floor function), endowed with a biinvariant metric $b$. Then $(G, b)$ has positive $S G B C$.

In particular, if $G$ is simple, then it has positive $S G B C$.

2. If $G / H$ is a normal homogeneous Riemannian manifold such that the rank $r$ of $G$ satisfies $r<\left[\frac{1}{2}(\operatorname{dim}(G / H)+1)\right]$, then $G / H$ has positive $S G B C$.

Corollary 3.4. If a compact manifold $M$ admits a smooth action of a compact connected simple Lie group with rank $r>\left[\frac{1}{2}(\operatorname{dim} M+1)\right]$, it admits a metric with positive $S G B C$.

\section{Proof of Theorem B}

Let $(M, g)$ and $(B, \check{g})$ be two Riemannian manifolds, and let $\pi:(M, g) \rightarrow(B, \check{g})$ be a Riemannian submersion. We define, for every $t \in \mathbb{R}$, a new Riemannian metric $g_{t}$ on the manifold $M$ by multiplying the metric $g$ by $t^{2}$ in the vertical directions. Recall that for all $m \in M$, we have a natural orthogonal decomposition of the tangent space at $m$ :

$$
T_{m} M=\mathscr{V}_{m} \oplus \mathscr{H}_{m},
$$

where $\mathscr{V}_{m}$ is the tangent to the fiber at $m$, and $\mathscr{H}_{m}$ is the horizontal space.

In this case, $\pi:\left(M, g_{t}\right) \rightarrow(B, \check{g})$ is still a Riemannian submersion with the same horizontal and vertical distributions; see [Besse 1987; Labbi 1997a].

We now index by $t$ all the invariants of the metric $g_{t}$, and in the case $t=1$, we omit the index 1 . We use a hat "^" to denote invariants of the fibers with the induced metric, and a check " " " to denote those of the basis $B$ ).

Using [Labbi 1997a, Lemma 2.1], it is easy to show that for all $g_{t}$-unit tangent vectors $e_{1}, e_{2}, e_{3}, e_{4}$, we have

$$
R_{t}\left(e_{1}, e_{2}, e_{3}, e_{4}\right)=O\left(\frac{1}{t}\right)
$$

if one of these vectors is horizontal, and

$$
R_{t}\left(e_{1}, e_{2}, e_{3}, e_{4}\right)=\frac{1}{t^{2}} \hat{R}\left(t e_{1}, t e_{2}, t e_{3}, t e_{4}\right)+O(1)
$$


if the four vectors are vertical. Consequently, if $\left\{e_{1}, e_{2}, \ldots, e_{n}\right\}$ is a $g_{t}$-orthonormal basis such that $\left\{e_{1}, \ldots, e_{q}\right\} \in \mathscr{V}_{m}$ and $\left\{e_{q+1}, \ldots, e_{n}\right\} \in \mathscr{H}_{m}$, then

$$
\begin{aligned}
\left\|R_{t}\right\|_{t}^{2} & =\sum_{\substack{1 \leq i<j \leq n \\
1 \leq k<l \leq n}} R_{t}\left(e_{i}, e_{j}, e_{k}, e_{l}\right)^{2} \\
& =\frac{1}{t^{4}} \sum_{\substack{1 \leq i<j \leq q \\
1 \leq k<l \leq q}} \hat{R}\left(t e_{i}, t e_{j}, t e_{k}, t e_{l}\right)^{2}+O\left(\frac{1}{t^{2}}\right)=\frac{1}{t^{4}}\|\hat{R}\|^{2}+O\left(\frac{1}{t^{2}}\right), \\
\left(\left\|\operatorname{Ric}_{t}\right\|_{t}\right)^{2} & =\sum_{\substack{1 \leq i \\
j \leq n}} \operatorname{Ric}_{t}\left(e_{i}, e_{j}\right)^{2}=\frac{1}{t^{4}} \sum_{\substack{1 \leq i \\
j \leq q}} \widehat{\operatorname{Ric}}\left(t e_{i}, t e_{j}\right)^{2}=\frac{1}{t^{4}}\|\widehat{\operatorname{Ric}}\|^{2}+O\left(\frac{1}{t^{2}}\right), \\
\left(\left\|\operatorname{scal}_{t}\right\|_{t}\right)^{2} & =\frac{1}{t^{4}}\|\widehat{\operatorname{scal}}\|^{2}+O\left(\frac{1}{t^{2}}\right) .
\end{aligned}
$$

Therefore, at the point $m$ we have

$$
\left(h_{4}\right)_{t}=\frac{1}{t^{4}} \hat{h}_{4}+O\left(\frac{1}{t^{2}}\right) .
$$

This completes the proof of Theorem B since the total space is compact.

Corollary 4.1. 1. The product $S^{p} \times M$ of an arbitrary compact manifold $M$ with a sphere $S^{p}$, where $p \geq 4$, admits a Riemannian metric with positive $S G B C$.

2. If a compact manifold admits a Riemannian foliation such that the leaves have positive $S G B C$, then the manifold admits a Riemannian metric with positive $S G B C$.

Proof. The first part is straightforward. To prove the second, it suffices to notice that the proof of Theorem B works also in the case of local Riemannian submersions.

Corollary 4.2. If a compact manifold $M$ admits a free and smooth action of a compact connected Lie group $G$, with rank $r<\left[\frac{1}{2}(\operatorname{dim} G+1)\right]$, then the manifold $M$ admits a Riemannian metric with positive $S G B C$.

Proof. The canonical projection $M \rightarrow M / G$ is in this case a smooth submersion. Let the fibers be equipped with a biinvariant metric from the group $G$ via the canonical inclusion $G \subset T_{m} M$.

Using any $G$-invariant metric on $M$, we define the horizontal distribution to which we lift up an arbitrary metric from the basis $M / G$. Thus we have defined a metric on $M$ such that the projection $M \rightarrow M / G$ is a Riemannian submersion.

Finally, since the group $G$ with a biinvariant metric has positive SGBC, then so are the fibers with the induced metric. and we conclude using Theorem B.

Remark. All simple Lie groups satisfy the property $r<\left[\frac{1}{2}(\operatorname{dim} G+1)\right]$. 


\section{Proof of Theorem C}

We proceed as in Gromov and Lawson's proof [1980] for the case of scalar curvature.

Let $(X, g)$ be a compact $n$-dimensional Riemannian manifold with positive SGBC, and let $S^{m} \subset X$ be an embedded sphere of codimension $q$, with trivial normal bundle $N \equiv S^{m} \times \mathbb{R}^{q}$. There exists $r_{0}>0$ such that the exponential map exp : $S^{m} \times D^{q}\left(r_{0}\right) \rightarrow X$ is an embedding, $\{x\} \times D^{q}\left(r_{0}\right)$, for $x \in S^{m}$, denotes the closed Euclidean ball in $\mathbb{R}^{q} \equiv\{x\} \times \mathbb{R}^{q}$. Let $\exp ^{*} g$ denote the pull back of the metric $g$ to the normal subbundle $S^{m} \times D^{q}\left(r_{0}\right)$.

Another natural metric on the normal bundle is the metric $g^{\nabla}$ defined using the normal connection $\nabla$, which is the metric compatible with the normal connection, such that the natural projection $\pi: S^{m} \times D^{q}(r) \rightarrow S^{m}$ is a Riemannian submersion. We shall denote also by $g^{\nabla}$ its restriction to the subbundles

$$
S^{m} \times D^{q}(r) \quad \text { and } \quad \partial\left(S^{m} \times D^{q}(r)\right)=S^{m} \times S^{q-1}(r) .
$$

Recall that at each $(p, v) \in S^{m} \times D^{q}(r)$ we have a natural $g^{\nabla}$-orthogonal decomposition of the tangent space into vertical and horizontal subspaces, namely,

$$
T_{(p, v)} S^{m} \times D^{q}(r)=\mathscr{V}_{(p, v)} \oplus \mathscr{H}_{(p, v)},
$$

where $\mathscr{V}_{(p, v)}$ is the tangent space to the fiber (over $p$ ) at $v$. Note that $\pi_{*}\left(\mathscr{V}_{(p, v)}\right)=$ $\{0\}$ and $\pi_{*}\left(\mathscr{H}_{(p, v)}\right)=T_{p} S^{m}$. These two metrics are tangent to the order two in the directions tangent to $D^{q}$, precisely for $u_{1}, u_{2} \in T_{(p, v)} S^{m} \times D^{q}(r)$. We have

$$
\exp ^{*} g\left(u_{1}, u_{2}\right)=g^{\nabla}\left(u_{1}, u_{2}\right)+\mathrm{II}_{n}\left(\pi_{*} u_{1}, \pi_{*} u_{2}\right) r+O\left(r^{2}\right)
$$

(see [Labbi 2006]), where $\mathrm{II}_{n}$ denotes the second fundamental form of $M$ at $p$, in the direction of the unit normal vector $n=v /\|v\|$.

Remark. In [Gromov and Lawson 1980, p. 430], at the beginning of the proof of Lemma 2, it is claimed that the former metrics are sufficiently close in the $C^{2}$ topology. But in general this is true only for the directions tangent to $S^{q-1}(r)$. The same error occurs also in [Labbi 1997b]. A detailed study of the behavior of these two metrics will appear in [Labbi 2006], but in brief, here is the situation:

With respect to the metric $g^{\nabla}$, the sphere $S^{m} \hookrightarrow S^{m} \times D^{q}$ is totally geodesic (since for a Riemannian submersion the horizontal lift of a geodesic is a geodesic). But on the other hand, the sphere $S^{m} \hookrightarrow S^{m} \times D^{q}$ is totally geodesic for the metric $\exp ^{*} g$ only if the sphere $S^{m}$ is totally geodesic in $(X, g)$.

However this does not affect the corresponding conclusions in both papers (after minor changes), since the curvatures in question (that is the scalar curvature and the $p$-curvatures, $p \leq q-3)$ of these two metrics on the bundles $S^{m} \times S^{q-1}(r)$ are high and close enough as $r \rightarrow 0$. 
It is easy to see that the matrix of the second fundamental form of $S^{m} \times S^{q-1}(r)$ in $S^{m} \times D^{q}(r)$, with respect to the decomposition (8), is of the form

$$
\left(\begin{array}{cc}
-\mathrm{Id} / r & 0 \\
0 & 0
\end{array}\right) \text {. }
$$

Thus, using formulas (9) and (10), one can deduce without difficulty that the matrix of the second fundamental form of $S^{m} \times S^{q-1}(r)$ in $S^{m} \times D^{q}(r)$, with respect to the metric $\exp ^{*} g$ (and the decomposition (8)) has the form

$$
\left(\begin{array}{cc}
-\mathrm{Id} / r+O(r) & O(r) \\
O(r) & O(1)
\end{array}\right) .
$$

The matrix of the second fundamental form still has the same form (11) with respect to the exp* $g$-orthogonal decomposition

$$
T_{(p, v)} S^{m} \times D^{q}(r)=\mathscr{V}_{(p, v)} \oplus \mathcal{H}_{(p, v)}^{\prime},
$$

where $\mathscr{V}_{(p, v)}$ is as in (8), and the distribution $\mathscr{H}^{\prime}$ is defined by the preceding orthogonal decomposition. As $r \rightarrow 0$, the distribution $\mathscr{H}^{\prime}$ converges to the distribution $\mathscr{H}$ defined by the decomposition (8).

Now we define a hypersurface $M$ in the product $S^{m} \times D^{q}\left(r_{0}\right)$, endowed with the product metric $\exp ^{*} g \times \mathbb{R}$, by the relation

$$
M=\left\{((x, v), t) \in S^{m} \times D^{q}\left(r_{0}\right) \times \mathbb{R}:(\|v\|, t) \in \gamma\right\},
$$

where $\gamma$ is a curve whose graph in the $(r, t)$-plane looking like this:

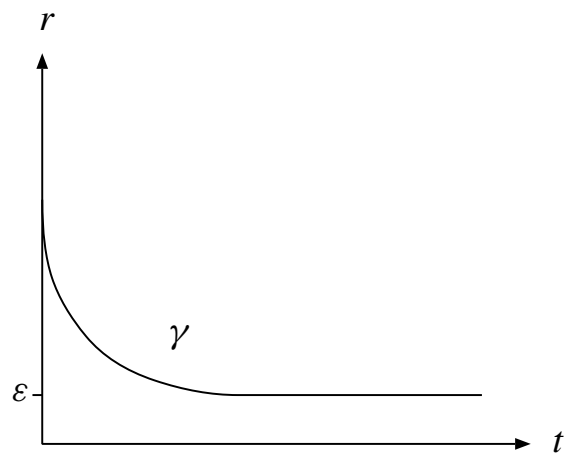

The important features of $\gamma$ are that it is tangent to the $r$-axis at $t=0$, and it is constant for $r=\varepsilon>0$. Thus the induced metric on $M$ extends the metric $\exp ^{*} g$ on $S^{m} \times D^{q}\left(r_{0}\right)$ near its boundary, and finishes with the product metric $\left(\partial\left(S^{m} \times D^{q}(\varepsilon)\right), \exp ^{*} g\right) \times \mathbb{R}=\left(S^{m} \times S^{q-1}(\varepsilon), \exp ^{*} g\right) \times \mathbb{R}$. 
Next, we evaluate the SGBC of the hypersurface $M$. For each $m \in M$, we have the $\exp ^{*} g$-orthogonal decomposition

$$
T_{m} M=\mathbb{R} \tau \oplus \mathscr{V}_{m} \oplus \mathcal{H}_{m}^{\prime},
$$

where $\tau$ is the unit tangent vector to the curve $\gamma$ in the $(r, t)$-plane, and $\mathscr{V}_{m}, \mathscr{H}_{m}^{\prime}$ are as in (12).

A straightforward computation using (11) shows that the matrix of the second fundamental form of the hypersurface $M$ has, with respect to the decomposition (13), the form

$$
\left(\begin{array}{ccc}
k & 0 & 0 \\
0 & & \\
& (-\mathrm{Id} / r+O(r)) \sin \theta & O(r) \sin \theta \\
\vdots & O(r) \sin \theta & O(1) \sin \theta
\end{array}\right),
$$

where $k$ denotes the curvature of the curve $\gamma$ in the $(r, t)$-plane, and $\theta$ denotes the angle between the normal to $M$ and the $t$-axis at the corresponding point.

Then a long but straightforward computation using the Gauss equation and the matrix (14) shows that the curvatures of $M$ have the form

$$
\begin{gathered}
\left\|R^{M}\right\|^{2}=\left\|R^{S^{p} \times D^{q}}\right\|^{2}+\frac{(q-1)(q-2)}{2 r^{4}} \sin ^{4} \theta+(q-1) \frac{k^{2}}{r^{2}} \sin ^{2} \theta+O\left(\frac{1}{r^{2}}\right) \sin \theta, \\
\left\|\mathrm{Ric}^{M}\right\|^{2}=\left\|\mathrm{Ric}^{S^{p} \times D^{q}}\right\|^{2}+\frac{(q-1)(q-2)^{2}}{r^{4}} \sin ^{4} \theta+q(q-1) \frac{k^{2}}{r^{2}} \sin ^{2} \theta \\
-\frac{(q-1)(q-2)^{2} k}{r^{3}} \sin ^{3} \theta+O\left(\frac{1}{r^{2}}\right) \sin \theta, \\
\left\|\mathrm{scal}^{M}\right\|^{2}=\left\|\mathrm{scal}^{S^{p} \times D^{q}}\right\|^{2}+\frac{(q-1)^{2}(q-2)^{2}}{r^{4}} \sin ^{4} \theta+4(q-1)^{2} \frac{k^{2}}{r^{2}} \sin ^{2} \theta \\
-2 \frac{(q-1)^{2}(q-2) k}{r^{3}} \sin ^{3} \theta+O\left(\frac{1}{r^{3}}\right) \sin \theta,
\end{gathered}
$$

where we have assumed that the curve $\gamma$ has curvature $k=O(1 / r)$.

Hence we can evaluate the SGBC of $M$ as follows:

$$
\begin{aligned}
h_{4}^{M}=h_{4}^{S^{p} \times D^{q}}+\frac{(q-1)(q-2)(q-3)(q-4)}{4 r^{4}} \sin ^{4} \theta & \\
- & \frac{(q-1)(q-2)(q-3) k}{2 r^{3}} \sin ^{3} \theta+O\left(\frac{1}{r^{3}}\right) \sin \theta .
\end{aligned}
$$

Next we show that it is possible to choose the curve $\gamma$ so that the metric induced on $M$ has positive SGBC at all points $m \in M$. 
Formula (15) shows that $h_{4}^{M}=h_{4}^{S^{p} \times D^{q}}$ is positive for $\theta=0$, and then there exists an angle $\theta_{0}>0$ such that, for all $0<\theta \leq \theta_{0}$, the SGBC of $M$ is positive.

Then we continue with a straight line $(k=0)$ of angle $\theta_{0}$, say $\gamma_{1}$, until the term in $\sin ^{4} \theta_{0}$ is strongly dominating.

On the other hand, when $\theta=\pi / 2$, then $k=0$ and $r=\epsilon$. We have

$$
h_{4}^{M}=\frac{(q-1)(q-2)(q-3)(q-4)}{4 \epsilon^{4}}+O\left(\frac{1}{\epsilon^{3}}\right),
$$

which is positive as $\epsilon$ is sufficiently small, and $q \geq 5$.

We now choose $r_{1}$ positive and small. Considering the point $\left(r_{1}, t_{1}\right) \in \gamma_{1}$, we bend the straight line $\gamma_{1}$, beginning at this point, with a curvature $k(s)$ of the form shown here, where $s$ denotes arc length along the curve.

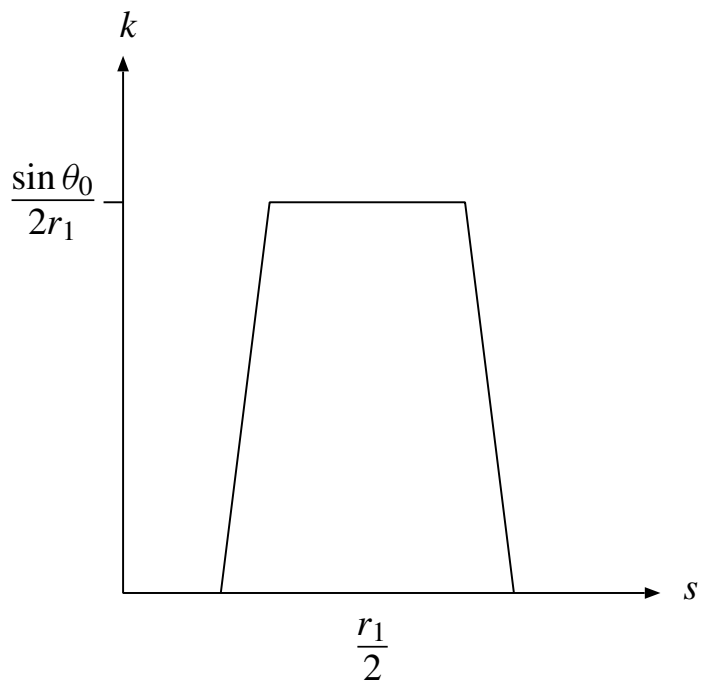

Since $q \geq 5$, formula (15) shows that

$$
h_{4}^{M} \geq h_{4}^{S^{p} \times D^{q}}+\frac{(q-1)(q-2)(q-3)}{2 r^{3}} \sin ^{3} \theta\left(\frac{\sin \theta}{2 r}-k\right)+O\left(\frac{1}{r^{3}}\right) \sin \theta .
$$

Then it is clear that the hypersurface $M$ will continue to satisfy $h_{4}^{M}>0$, since $k<\left(\sin \theta_{0} / 2 r_{1}\right)<(\sin \theta / 2 r)$.

After this first bending, we have $\Delta r \leq \Delta s=r_{1} / 2$, and then $r \geq r_{1}-\Delta r \geq$ $r_{1}-\left(r_{1} / 2\right)>0$. Consequently the curve will not cross the $t$-axis.

On the other hand, $\Delta \theta=\int k d s \approx \sin \theta_{0} / 4$ is independent of $r_{1}$. Clearly, by scaling down the curvature $k$, we can produce any $\Delta \theta$ such that $0<\Delta \theta \leq \sin \theta_{0} / 4$.

Our curve now continues with a new straight line $\gamma_{2}$ with angle $\theta_{1}=\theta_{0}+\Delta \theta$. By repeating this process finitely many times we can achieve a total bend of $\pi / 2$. 
Let $g_{\epsilon}$ denote the induced metric from $\exp ^{*} g$ on $\partial\left(S^{p} \times D^{q}(\epsilon)\right)=S^{p} \times S^{q-1}(\epsilon)$, and recall that the new metric defined on $M$ is the old metric when $t=0$, and finishes with the product metric $g_{\epsilon} \times \mathbb{R}$.

We will now deform the product metric $g_{\epsilon} \times \mathbb{R}$ on $S^{p} \times S^{q-1}(\epsilon)$ to the standard product metric, through metrics with positive SGBC. This is done in two steps:

Step 1: We deform the metric $g_{\epsilon}$ on $S^{m} \times S^{q-1}(\epsilon)$ to the standard product metric $S^{m}(1) \times S^{q-1}(\epsilon)$ through metrics with positive SGBC, as follows:

First, the metric $g_{\epsilon}$ can be homotoped through metrics, with $h_{4}>0$, to the normal metric $g^{\nabla}$, since their SGBC are respectively high and close enough; see (16) and (7).

Then, for $\epsilon$ small enough, we can deform the normal metric $g^{\nabla}$ on $S^{m} \times S^{q-1}(\epsilon)$ through Riemannian submersions to a new metric, where $S^{p}$ is the standard sphere $S^{p}(1)$, keeping the horizontal distribution fixed.

This deformation keeps $h_{4}>0$ as long as $\epsilon$ is small enough; see (7).

Finally, we deform the horizontal distribution to the standard one, and again by the same formula (7), this can be done keeping $h_{4}>0$.

Step 2: Let us denote by $d s_{t}^{2}$, for $0 \leq t \leq 1$, the previous family of deformations on $S^{m} \times S^{q-1}(\epsilon)$. They all have positive SGBC, where $d s_{0}=g_{\epsilon}$, and $d s_{1}$ is the standard product metric.

It is clear that the metric

$$
d s_{t / a}^{2}+d t^{2}
$$

for $0 \leq t \leq a$, glues together the two metrics $d s_{0} \times \mathbb{R}$ and $d s_{1} \times \mathbb{R}$. Furthermore, there exists $a_{0}>0$ such that, for all $a \geq a_{0}$, the metric

$$
d s_{t / a}^{2}+d t^{2}
$$

on $S^{m} \times S^{q-1}(\epsilon) \times[0, a]$ has positive SGBC. In fact, via a change of variable, this is equivalent to the existence of $\lambda_{0}>0$ such that, for all $0<\lambda \leq \lambda_{0}$, the metric $\lambda^{2} d s_{t}^{2}+d t^{2}$ has positive SGBC. This is already known to be true, again by formula (7). This completes the proof of the theorem.

Corollary 5.1. Let $G$ be a finitely presented group. Then for every $n \geq 6$, there exists a compact n-manifold $M$ with positive $S G B C$ such that $\pi_{1}(M)=G$.

Proof. Let $G$ be a group with presentation consisting of $k$ generators $x_{1}, x_{2}, \ldots$, $x_{k}$, and $l$ relations $r_{1}, r_{2}, \ldots, r_{l}$.

Let $S^{1} \times S^{n-1}$ be endowed with the standard product metric, which has positive SGBC (we have $n-1 \geq 4$ ). Note that the fundamental group of $S^{1} \times S^{n-1}$ is infinite cyclic. Hence by taking the connected sum $N$ of $k$ copies of $S^{1} \times S^{n-1}$, we obtain an orientable compact $n$-manifold with positive SGBC (since this operation 
is a surgery of codimension $n \geq 5$ ). By the van Kampen theorem, the fundamental group of $N$ is a free group on $n$ generators, which we denote by $x_{1}, x_{2}, \ldots, x_{k}$.

We now perform surgery $l$ times on the manifold $N$, killing in succession the elements $r_{1}, r_{2}, \ldots, r_{l}$. The result is a compact, orientable $n$-manifold $M$, with positive SGBC (since the surgery is of codimension $n-1 \geq 5$ ), such that $\pi_{1}(M)=$ $G$, as desired.

\section{References}

[Besse 1987] A. L. Besse, Einstein manifolds, vol. 10, Ergebnisse der Mathematik und ihrer Grenzgebiete (3) [Results in Mathematics and Related Areas (3)], Springer, Berlin, 1987. MR 88f:53087 Zbl 0613.53001

[Gromov and Lawson 1980] M. Gromov and H. B. Lawson, Jr., "The classification of simply connected manifolds of positive scalar curvature", Ann. of Math. (2) 111:3 (1980), 423-434. MR 81h: 53036 Zbl 0463.53025

[Kulkarni 1972] R. S. Kulkarni, “On the Bianchi identities”, Math. Ann. 199 (1972), 175-204. MR 49 \#3767 Zbl 0234.53021

[Labbi 1997a] M.-L. Labbi, "Actions des groupes de Lie presque simples et positivité de la $p$ courbure”, Ann. Fac. Sci. Toulouse Math. (6) 6:2 (1997), 263-276. MR 99e:53052 Zbl 0898.53032

[Labbi 1997b] M.-L. Labbi, "Stability of the $p$-curvature positivity under surgeries and manifolds with positive Einstein tensor", Ann. Global Anal. Geom. 15:4 (1997), 299-312. MR 98e:53065 Zbl 0888.53033

[Labbi 2000] M.-L. Labbi, "On compact manifolds with positive isotropic curvature", Proc. Amer. Math. Soc. 128:5 (2000), 1467-1474. MR 2000j:53049 Zbl 0948.53017

[Labbi 2005] M.-L. Labbi, "Double forms, curvature structures and the $(p, q)$-curvatures", Trans. Amer. Math. Soc. 357:10 (2005), 3971-3992. MR 2006g:53039 Zbl 1077.53033

[Labbi 2006] M.-L. Labbi, "On two natural metrics on a tubular neighborhood of an embedded submanifold”, Preprint, 2006. math.DG/0607523

[Thorpe 1969] J. A. Thorpe, "Some remarks on the Gauss-Bonnet integral", J. Math. Mech. 18 (1969), 779-786. MR 41 \#963 Zbl 0183.50503

Received February 8, 2005. Revised June 15, 2005.

MOHAMMED-LARBi LABBi

DEPARTMENT OF MATHEMATICS

COLLEGE OF SCIENCE

UNIVERSITY OF BAHRAIN

32038 ISA TOWN

BAHRAIN

labbi@sci.uob.bh 